\title{
A robust method for the rapid detection of microsatellite instability in colorectal cancer
}

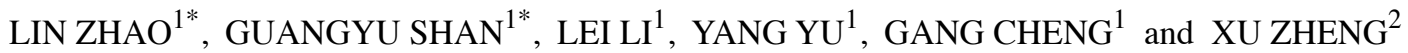 \\ ${ }^{1}$ Beijing Novogene Bioinformatics Technology Co., Ltd, Beijing 100015; ${ }^{2}$ Department of Oncology, \\ Shengjing Hospital of China Medical University, Shenyang, Liaoning 110004, P.R. China
}

Received April 26, 2019; Accepted January 23, 2020

DOI: $10.3892 / \mathrm{ol} .2020 .11702$

\begin{abstract}
Although several computational tools using next-generation sequencing (NGS) data have been proposed to detect microsatellite instability (MSI) status, they still have limitations and need improvement. We developed a NovoPM-MSI method to detect MSI status based on NGS data. This method evaluated target mononucleotide microsatellite loci that were sequenced during targeted gene enrichment analysis and reported sample instability score as the fraction of unstable loci within the target set after assessing locus instability by comparing length distribution in paired tumor-normal samples. We validated this method against the conventional MSI-PCR method in 113 paired colorectal cancer (CRC) specimens and compared the performance of NovoPM-MSI to that of mSINGS and MANTIS in accuracy and runtime efficiency. By using the MSI status from MSI-PCR as the gold standard, the three computational methods showed the same sensitivity of $88.9 \%$ but different specificities (NovoPM-MSI 97.1\%, MANTIS $86.5 \%$ and mSINGS $99.0 \%$ ). Only NovoPM-MSI could greatly improve both the sensitivity and specificity by setting an ambiguous interval. MANTIS had the shortest average runtime $(16.3 \mathrm{sec})$, followed by NovoPM-MSI $(18.3 \mathrm{sec})$ and mSINGS $(109.0 \mathrm{sec})$. In short, the NovoPM-MSI method provides a fast and reliable MSI detection method with accuracy comparable to MSI-PCR in paired CRC samples.
\end{abstract}

\section{Introduction}

Numerous guidelines recommend tumor screening via microsatellite instability (MSI) analysis in all patients with colorectal cancer (CRC), as MSI status has substantial

Correspondence to: Dr Xu Zheng, Department of Oncology, Shengjing Hospital of China Medical University, 36 Sanhao Street, Heping, Shenyang, Liaoning 110004, P.R. China

E-mail: xuzheng_7722@163.com

*Contributed equally

Key words: bioinformatics, colorectal, computational tools, microsatellite instability, next-generation sequencing implications in CRC diagnosis (1), prognosis (2) and responses to fluorouracil (3). Fast progress in cancer immunotherapy also confirmed the good response of MSI tumors to checkpoint inhibitor blockade $(4,5)$. Recently, the US Food and Drug Administration (FDA) approved pembrolizumab as the first drug for any solid tumor with MSI-high status (https://www.fda. gov/newsevents/newsroom/pressannouncements/ucm560167. $\mathrm{htm}$ ); the clinical detection of MSI status is in great demand for patients with CRC or other cancer types.

MSI status is a molecular fingerprint for DNA mismatch repair (MMR) system deficiency. Dysfunctional MMR genes (MLH1, MSH2, MSH6 and PMS2) may introduce length-altering mutations in microsatellites (MS), which are short tandem repeat sequences dispersed throughout the human genome and could be used as deficient MMR (dMMR) markers $(6,7)$. Two of the most common methods for MSI detection have been developed and are still considered the gold standards. MSI-PCR assesses length variability at several standardized MS loci, while immunohistochemical (IHC) staining inspects the protein expression of MMR genes. High frequency MSI (MSI-H) status is determined by the fraction of unstable MS markers (MSI-PCR) or the loss of one or more MMR proteins (IHC). However, considering the various outcomes of dMMR, these traditional methods may have limitations. The indirect IHC method cannot detect all abnormalities of MMR genes and depends heavily on specialized techniques (8). A recent study demonstrated that 5-marker MSI-PCR had inferior sensitivity in 91 prostate tumors among other methods with more markers (9).

With the drastic development of next-generation sequencing (NGS) technologies, the clinical management of cancer patients has been facilitated in a number of ways $(10,11)$. A prominent advantage of NGS detection is that sufficient genetic information can be obtained with much lower costs than low-throughput methods by well-designed tumor sequencing. Therefore, more MS loci could simultaneously be assessed by NGS sequencing to provide new possibilities to evaluate MSI status. Recently, several computational methods for MSI detection, which can be integrated into existing NGS pipelines, have been designed and proposed to provide comprehensive information on tumor genomics. By comparing tumor DNA sequences to normal DNA sequences, such methods can infer MSI status according to either tumor mutation burden (12-14) or read count distribution. Two typical approaches based on 
read count distribution are mSINGS (15) and MANTIS (16). These methods evaluate each MS locus within a target marker set and report the MSI status by specifying certain cut-offs. However, these approaches differ in their determination of tumor instability. mSINGS compares tumor-only samples to a pre-constructed baseline-control and evaluates tumor instability by the fraction of unstable MS loci within a target set. By contrast, MANTIS compares each target locus in both tumor and matched healthy samples and calculates tumor instability as the averaged stepwise difference across all target loci. These two methods might have disadvantages due to their algorithms. mSINGS expends more time to analyze one sample for the pileup step and statistical test for every locus. However, since MANTIS does not employ a statistical test to calculate the MSI score, this method may be weak at exploiting informative MS loci and may lead to biased results.

Herein, a robust and rapid algorithm called NovoPM-MSI was developed by using a targeted tumor-sequencing panel to reliably assess the MSI status in CRC patients. The read count distribution from paired tumor-healthy samples was used to develop an improved strategy in order to determine tumor instability by examining target MS loci. We also validated this algorithm against conventional MSI-PCR in 113 CRC cases. Finally, we demonstrated the high performance of NovoPM-MSI over mSINGS and MANTIS in MSI status detection and runtime efficiency.

\section{Materials and methods}

Sample source and next-generation sequencing. To develop the NovoPM-MSI algorithm, retrospective CRC specimens from 113 patients were utilized. The study was conducted in accordance with the Declaration of Helsinki and the protocol was approved by the Ethics Committee of Shengjing Hospital of China Medical University. All the recruited patients had signed informed consent for their samples to be used in the study and all clinical data and specimens were received anonymously. These specimens were previously analyzed in our CAP-certified laboratory (Tianjin Novogene Med LAB). All cases were tumor tissue DNA extracted from formalin-fixed paraffin-embedded (FFPE) specimens with matched white blood cell (WBC) DNA. These paired cases were sequenced using a targeted gene panel, NovoPM, between January 2016 and October 2017. This panel covers all coding and 21 non-coding regions of the 548 genes associated with multiple tumor types. In the sequencing assay, DNA from tumor and matched WBCs was used to construct sequencing libraries by a hybrid-capture selection method. These libraries were sequenced with a highly uniform depth (targeting $>1,000 \mathrm{X}$ coverage by non-PCR duplicated read pairs) on the Illumina Hi-Seq X Ten platform as paired-end reads. The protocols and reagents have been optimized to ensure uniform coverage and robust performance for a wide variety of specimens.

Selection of microsatellite marker loci. To identify MS repeats across the human genome with high confidence, a reference FASTA file (hg19/GRCh37) was first scanned using an in-house pipeline. A qualified MS locus was defined as follows: i) The base number within a microsatellite locus should range from 10 to 100 ; and ii) the minimum length of each motif should be
5. The total number of MS loci across the reference genome was $2,946,833$. The mononucleotide repeats covered by the NovoPM panel were extracted from the MS loci in order to identify MS markers for MSI detection among those repeats. Only mononucleotide repeats were selected as MS markers, since these sequences are more sensitive in traditional MSI detection.

Workflow of NovoPM-MSI. A brief illustration of the NovoPM-MSI detection workflow is presented in Fig. 1. During the data pre-processing stage, the sequencing reads obtained after the in-house QC pipeline assessment were aligned using the Burrows-Wheeler Aligner (version 0.7.8) (17) against the reference genome (hg19/GRCh37) and indexed by SAMtools (version 0.1.19) (18). Duplicate reads were removed using Picard Tools (version 1.96) (http://broadinstitute.github. io/picard/) from BAM files. A reference genome and target loci were required as well as paired tumor-normal BAM files in NovoPM-MSI.

Low-quality reads were removed step by step if they met the following conditions: i) reads from paired tumor-normal BAM files that were either too short $(<35 \mathrm{bp})$ or had low average base quality $(<25.0)$; ii) reads covering the MS locus that had delimited length and lower average base quality $(<30.0)$; and iii) reads with no clipped parts that were shorter than a second length threshold $(<35)$. At each MS locus, minimum coverage was required in both tumor and normal samples $(>30 \mathrm{X})$ and each repeat type for an MS locus should have at least three qualified reads to support that type. A repeat length that was too long ( $>3 \mathrm{x}$ the standard deviation from mean) was defined as an outlier and was not considered in the subsequent steps.

Qualified supporting reads were then normalized by the average sequencing depth for the tumor and normal samples, prior to determining the instability for each MS locus. In NovoPM-MSI, the length distribution at each MS locus was characterized by the normalized supporting reads in tumor and normal samples. For example, for one MS locus with X number of repeat types, two vectors were obtained by counting the supporting reads for each repeat type in the normal and tumor samples, given as $\mathrm{Ni}$ and $\mathrm{Ti}(\mathrm{i}=1,2,3, \ldots, \mathrm{X})$, respectively. This locus was determined as unstable if the two vectors were significantly different by using the non-parametric Mann-Whitney U test $(\mathrm{P}<0.05)$.

Finally, an instability score was calculated by the fraction of unstable loci within the target MS markers. An empirical cut-off was set as 0.2 based on the guidelines for defining MSI positivity $(15,19)$.

MSI status determination by PCR. All the paired tumor-normal CRC samples were tested by the MSI-PCR method to obtain the standard MSI status. The PCR panel included six mononucleotide repeat markers (NR21, BAT26, NR27, BAT25, NR24 and MONO27) for MSI testing and three other markers (Penta C, Penta D and Amelogenin) for the sample contamination control. A fluorescence profile of amplified microsatellite DNA from paired samples was produced by capillary fluorescence electrophoresis (ABI 3730xl Genetic Analyzer, Applied Biosystems). If fluorescence peaks were present at any marker in the tumor sample but absent in the normal sample, then that marker was determined as unstable. MSI-H samples had two 


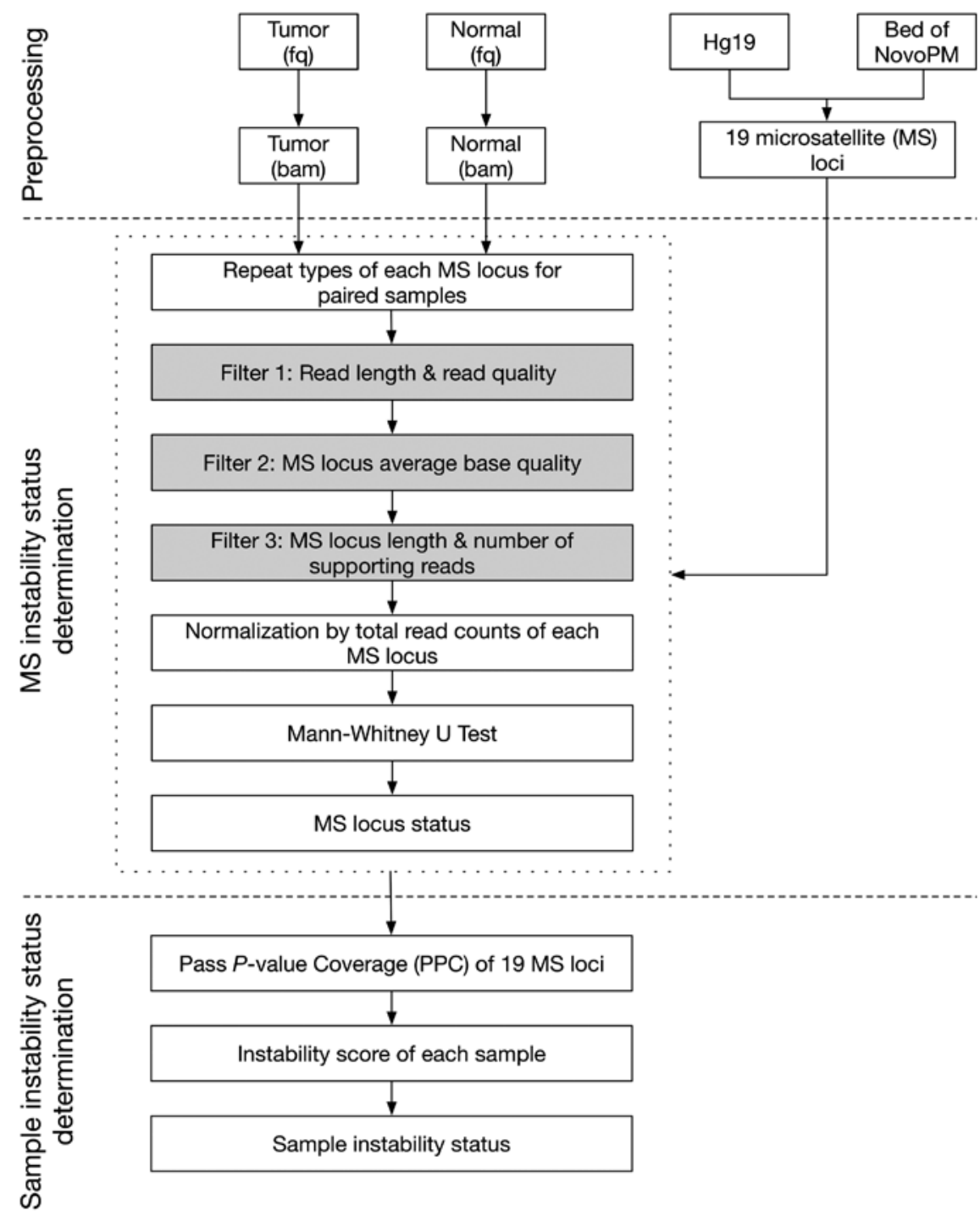

Figure 1. Overview of the NovoPM-MSI pipeline. Microsatellite (MS) loci are mononucleotide repeats loci screened from the NovoPM target region (Bed file). The per-locus read length distribution for the repeat types are collected from tumor and normal bam files. Supporting reads are filtered by minimum read length and minimum average base quality, whereas MS locus is filtered by minimum average base quality, abnormal locus length (mean $\pm 3 * \mathrm{SD}$ ) and the minimum supporting reads for locus repeat types. The supporting read counts at each MS locus are then normalized for paired samples and a Mann-Whitney $\mathrm{U}$ Test between each distribution is performed. Finally, the pass P-value coverage (PPC) is defined as the instability score for paired samples.

or more unstable markers, while MSI-L and MSS samples had one or zero unstable markers. Thus, the cut-off for the MSI-PCR method was $33.3 \%(2 / 6)$.

MSI detection by mSINGS and MANTIS. For mSINGS, a baseline control was first constructed by using 26 WBC samples from randomly selected MSI-negative cases. All the tumor samples were then tested by mSINGS, with defaults. The software determined an unstable locus by using the Z-test if the number of the types of repeat lengths was larger than (reference mean $+3 * \mathrm{SD}$ ), where the reference mean and SD were from the baseline-control. The software reported the percentage of unstable loci, according to which MSI-H samples were determined if the percentage was higher than $20 \%$ (15).

Additionally, all the paired samples were tested by MANTIS, which required paired tumor-healthy samples and a target marker set. The input files for MANTIS were the same as those required for NovoPM-MSI. MANTIS calculates the absolute value of the stepwise difference between tumor and healthy samples at each target MS locus using supporting read counts. Then, an average distance value across all the targeted loci was obtained to present the tumor instability. The samples were considered MSI-H when the average distance value was $>0.4(16)$

Performance comparison among three NGS-based tools. Three computational tools, NovoPM-MSI, MANTIS and mSINGS, were tested, with all samples having a standard MSI status assigned by MSI-PCR. Four performance indices, sensitivity (SN), specificity (SP), accuracy (ACC) and Mathew's correlation coefficient (MCC), were used to evaluate the detection performance among these tools. All the indices were calculated by convention.

Runtime efficiency was also tested by evaluating the processing time for each sample. Runtime data were compared by tools using pair-wise comparisons and the significance was adjusted by Bonferroni's correction (Welch's t-test, adjusted $\alpha=0.017)$. 
Table I. Microsatellite markers used in NovoMSI detection.

\begin{tabular}{|c|c|c|c|c|c|}
\hline Chr & Start & End & Function & Gene & Type \\
\hline 1 & 161309452 & 161309480 & Intronic & $S D H C$ & (T) 28 \\
\hline 3 & 12649448 & 12649472 & Intronic & $R A F 1$ & (A) 24 \\
\hline 3 & 37067099 & 37067120 & Intronic & MLH1 & (T) 21 \\
\hline 3 & 185802071 & 185802094 & Intronic & ETV5 & (A) 23 \\
\hline 4 & 55598211 & 55598236 & Intronic & KIT & (T) 25 \\
\hline 6 & 135527343 & 135527365 & Intronic & $M Y B$ & (A) 22 \\
\hline 7 & 140498359 & 140498380 & Intronic & $B R A F$ & (T) 21 \\
\hline 7 & 140508151 & 140508177 & Intronic & $B R A F$ & (A) 26 \\
\hline 8 & 38303723 & 38303746 & Intronic & $F G F R I$ & (T) 23 \\
\hline 8 & 48732074 & 48732095 & Intronic & $P R K D C$ & (A) 21 \\
\hline 9 & 5062500 & 5062531 & Intronic & $J A K 2$ & (A) 31 \\
\hline 9 & 87357704 & 87357731 & Intronic & NTRK2 & (T) 27 \\
\hline 9 & 87479651 & 87479673 & Intronic & NTRK2 & (T) 22 \\
\hline 12 & 11962746 & 11962772 & ncRNA_intronic & ETV6 & (T)26 \\
\hline 13 & 49039094 & 49039118 & Intronic & $R B 1$ & (T) 24 \\
\hline 15 & 99192754 & 99192778 & ncRNA_exonic & $I R A I N / I G F 1 R$ & (T) 24 \\
\hline 17 & 29559061 & 29559087 & Intronic & $N F 1$ & (T)26 \\
\hline 21 & 42863078 & 42863102 & Intronic & TMPRSS2 & (T) 24 \\
\hline $\mathrm{X}$ & 123195593 & 123195618 & Intronic & STAG2 & (T) 25 \\
\hline
\end{tabular}

\section{Results}

Target MS marker of NovoPM-MSI. A total of 19 MS loci of mononucleotide repeats located in non-coding regions were finally selected as the target marker set. These sensitive loci were sequenced after targeted gene enrichment during the NovoPM assay. Detailed information regarding these targets is listed in Table I.

Validation of three computational methods against conventional MSI-PCR. Among the 113 paired CRC tumor-normal cases, nine samples were assigned MSI-H and 104 samples were assigned MSS according to the gold standard of MSI-PCR. The fraction of MSI-H cases in the present study collection was $7.9 \%$, which was slightly lower than the number of MSI-H cases in the larger CRC population (15-20\%) $(20,21)$.

The initial cut-offs to determine the MSI-H status for NovoPM-MSI, MANTIS and mSINGS were set at 0.2, 0.2 and 0.4 , respectively. The validation of these methods against the MSI-PCR method is listed in Table II. All three computational methods correctly detected eight positive cases and one false negative case, which were not same for each tool, thus reaching the same sensitivity of $88.9 \%$. These analyses differed in specificity with descending order of mSINGS $(103 / 104$, 99\%), NovoPM-MSI (101/104, 97.1\%) and MANTIS (90/104, $86.5 \%$ ). According to Matthew's correlation coefficient, a more balanced measure, the performance of NovoPM-MSI (0.786) was slightly inferior to that of mSINGS (0.879), with MANTIS (0.516) showing the worst performance.

Detection comparison among NovoPM-MSI, MANTIS and $m S I N G S$. By plotting the instability score against samples, the detection performance among these computational tools was further assessed. NovoPM-MSI showed the least fluctuations within either the MSI-H (Fig. 2, left side of vertical line) or MSS category (Fig. 2, right side of vertical line). Although mSINGS had the highest MCC (0.879, Table II), the two misclassified samples were far from the default cut-off (0.2).

MANTIS showed the worst performance, with an accuracy of $86.7 \%$ (98/113) for the 14 false-positive samples that were reported (default cut-off: 0.4 ) and an acute fluctuate instability score produced.

In addition, the steady decrease in the instability score reported by NovoPM-MSI across MSI-H and MSS samples suggested an intermediate status for the MSI phenotype. We then modified a calling strategy of NovoPM-MSI by setting an ambiguous interval for the instability score. The small range was optimized by current observations set from 0.17 to 0.23 to include four samples with 'false' result. The samples were explicitly assigned MSI-H or MSS if the instability scores reported by NovoPM-MSI were $>0.23$ or $<0.17$. The samples were assigned MSI-ambiguous if the instability score fell into the ambiguous interval and these samples were recommended for further MSI-PCR testing. By setting the ambiguous interval, NovoPM-MSI reached $100 \%$ sensitivity and specificity. However, such intervals could not be properly utilized to improve the sensitivity and specificity of mSINGS.

Runtime comparison among NovoPM-MSI, MANTIS and $m S I N G S$. In addition to the accuracy of detection, the runtime for each sample is also important when evaluating tool performance. The ranking of runtime efficiency was MANTS (16.3 sec)>NovoPM-MSI (18.3 sec)>mSINGS (109.0 sec). All the pair-wise comparisons were highly significant among the three tools (Welch's t-test, $\mathrm{P}<0.01$, Fig. 3). 
Table II. Performance comparison among NovoMSI, MANTIS and mSINGS, using microsatellite instability-PCR results as the gold standard.

\begin{tabular}{lcrrrrrrr}
\hline Method & TP & TN & FP & FN & SN $(\%)$ & SP $(\%)$ & ACC (\%) & MCC \\
\hline NovoPM-MSI & 8 & 101 & 3 & 1 & 88.90 & 97.10 & 96.50 & 0.786 \\
MANTIS & 8 & 90 & 14 & 1 & 88.90 & 86.50 & 86.70 & 0.516 \\
mSINGS & 8 & 103 & 1 & 1 & 88.90 & 99.00 & 98.20 & 0.879 \\
\hline
\end{tabular}

TP, true-positive; TN, true-negative; FP, false-positive; FN, false-negative; SN, sensitivity; SP, specificity; ACC, accuracy; MCC, Matthew's correlation coefficient.

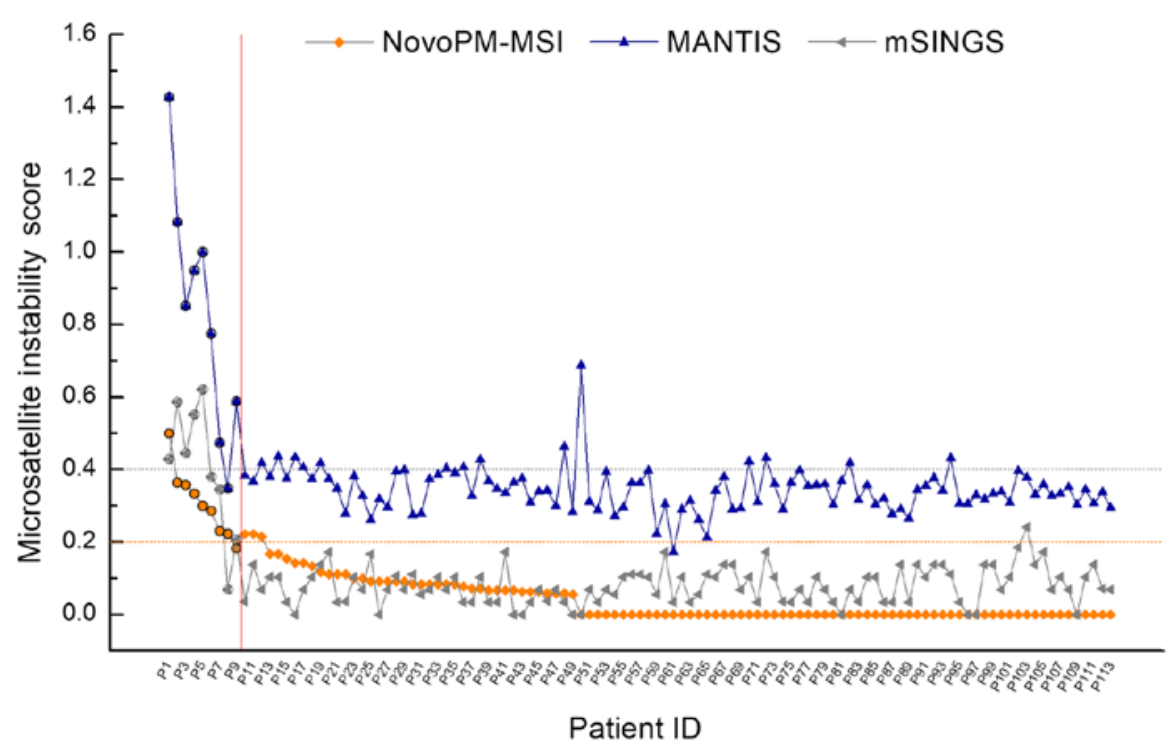

Figure 2. Microsatellite instability (MSI) scores from NovoPM-MSI (diamond), MANTIS (up triangle) and mSINGS (left triangle) for 113 CRC cases. The vertical line delimits all samples into MSI-high (left part) and MSS (right part) groups. The nine MSI-high samples determined by MSI-PCR are circled in black. The two horizontal lines indicate default cut-offs as references, with 0.2 for NovoPM-MSI and mSINGS and 0.4 for MANTIS. NovoPM-MSI shows the least fluctuations across the whole range of MSI status.

\section{Discussion}

In the present study, we described an NGS-based strategy, NovoPM-MSI, for MSI detection in paired tumor-healthy samples. We selected 19 mononucleotide MS repeats as target loci (Table I) since several studies have shown that mononucleotide repeats are the most sensitive in MSI detection (22-24). This new method evaluates each of the target MS loci by comparing the length distribution between tumor and normal samples and then calculates the percentage of unstable MS loci and reports the instability score as well as the MSI status according to empirical cut-offs. NovoPM-MSI has comparable reliability to the gold standard MSI-PCR, with robust performance (Table II).

We also compared NovoPM-MSI to two other NGS-based MSI detection tools, mSINGS (15) and MANTIS (16). All three tools exploit information from the read count distribution but differ in the instability evaluation. MANTIS assesses step-wise differences between tumor and matched normal samples at the same MS locus and calculates an average difference across all the MS loci to reflect the general instability of a sample but not a single locus. Notably, the aggregate averaged distance may be biased when using relatively fewer markers. One advantage of MANTIS is that this approach is less susceptible to sequencing

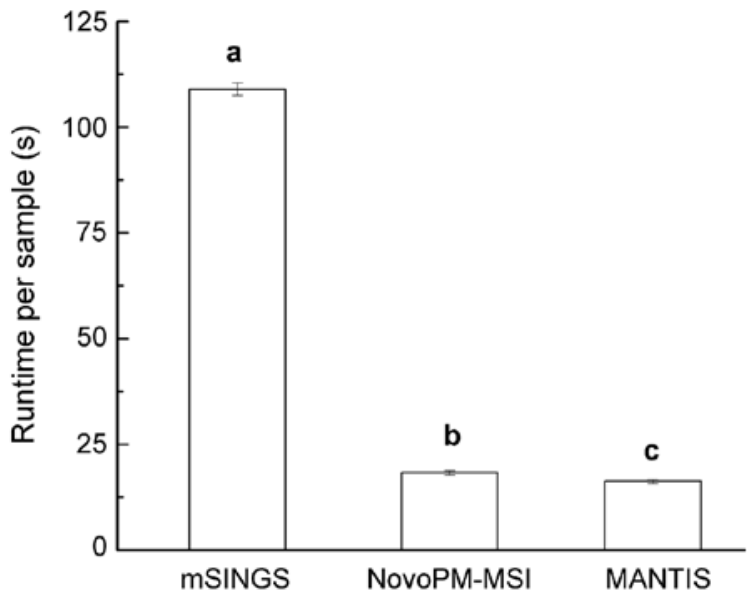

Figure 3. Comparison of the runtime efficiency among mSINGS, MANTIS and NovoPM-MSI $(n=113)$. Data show the mean and standard error of the mean. Letters a, b, and c above the error bars represent three comparison groups. Data with different letters are significantly different (Welch's t-test, $\mathrm{P}<0.01)$. The significance level after Bonferroni's correction is 0.017.

errors or poorly performing loci, but this advantage becomes increasingly apparent when using more target loci, which may 
be limited in most gene panels. In the present study, MANTIS produced the most false-positive calls (14 in 104) and showed the most fluctuations in the instability score (Table II and Fig. 2). mSINGS utilizes incidentally sequenced MS loci and evaluates the instability of each MS locus by comparison with a reference distribution generated from pooled normal samples. This approach assesses single locus instability using the Z-score test and reports the percentage of unstable loci as an instability score. mSINGS performed well for the 113 cases in the present study, with both high sensitivity (88.9\%) and specificity (99\%). However, its disadvantage in runtime, which was nearly six times longer than that of MANTIS, was also evident (Fig. 3). Additionally, the two misclassified samples could not be saved through setting an ambiguous interval. By contrast, NovoPM-MSI combines the advantages of the above tools. First, this approach examined an intermediate marker set (Table I), which was customized for most gene panels. Second, NovoPM-MSI runs much faster than mSINGS (18.3 vs. $109.0 \mathrm{sec}$ ) and has a runtime comparable to that of MANTIS $(16.3 \mathrm{sec})$. Finally, by setting an ambiguous interval (0.17-0.23) from empirical observations, the detection performance of NovoPM-MSI was greatly improved compared with that of the other tools. In short, NovoPM-MSI efficiently detected MSI status in a robust and rapid manner.

The advantages of NGS-based methods for MSI detection over traditional methods are obvious. MSI status can be simultaneously available with other genomic alterations, including SNV, gene fusion and indels, by NGS pipelines, without dedicated MSI detection. In addition, NGS-based methods enable the examination of more MS loci than that of the MSI-PCR method, providing a comprehensive assessment of MSI status. Although considered the gold standard, MSI-PCR reportedly shows $97 \%$ sensitivity and $95 \%$ specificity (25). In the present study, the three NGS-based methods showed performances comparable to those of conventional PCR (Table II). Recent studies have suggested that the MSI-H phenotype should be subdivided to guide important clinical applications (15,26-28). The rough binary classification of MSI only provides qualitative results and cannot meet this requirement. Alternatively, NGS-based methods can fill the gap by reporting both qualitative status and quantitative instability score. The NovoPM-MSI method reported in the present study showed reasonable classification (Fig. 2).

The present study may have some limitations. The NovoPM-MSI method may be limited by the small number of MSI-H cases (9/113), primarily due to the low frequency of MSI-H phenotypes in CRCs driven by MMR deficiency $(\sim 15 \%)(20,21)$. The accuracy of NovoPM-MSI would be increased if more MSI-H cases were accumulated, regardless of the one or two cut-offs used. We are now endeavoring to collect more CRC cases with different MSI status to build large validation cohort for NovoPM-MSI. In addition, using white blood cells as a normal control is relatively easy, but in most clinical settings, only tumor samples are available (e.g., retrospective FFPE without matched normal specimen). Under such conditions, tumor-only methods, including mSINGS, are required. In addition, MSI status is most closely studied in CRCs; it is found to be present in endometrial, ovarian, cervical, gastric and prostate cancers (27). The current NovoPM-MSI method has been developed for CRCs, but its applicability to other cancers may be tested in the future if cases from more cancer types are accumulated.
In conclusion, findings of the present study show that NovoPM-MSI can rapidly detect MSI status with high accuracy and robustness compared to conventional MSI-PCR and other NGS methods based on read count distributions. As the genetic characteristics of tumors become increasingly more critical in the clinical management of patients, we hope that the NovoPM-MSI method will provide more valuable information in clinical service when conducting tumor sequencing.

\section{Acknowledgements}

The authors would like to thank Ms Wanchun Zang, an experienced analyst for clinical data (Beijing Novogene Bioinformatics Technology Co. Ltd.), for her helpful suggestions on the manuscript.

\section{Funding}

This work was supported by grants from the research program of Novogene Bioinformatics Technology Co., Ltd. (grant no. P101ZY17010010).

\section{Availability of data and materials}

The datasets used and/or analyzed during the current study are available from the corresponding author on reasonable request.

\section{Authors' contributions}

LZ, GS, LL, GC and XZ designed the study. LZ and GS improved the algorithm, analyzed and interpreted all the data. LZ, GS, LL and GC wrote the manuscript. YY contributed to sample collection and project coordination. GC and $\mathrm{XZ}$ provided the research materials, supervised the study and edited the manuscript. All authors read and approved the final version of the manuscript.

\section{Ethics approval and consent to participate}

The present study was approved by the Ethics Committee of Shengjing Hospital of China Medical University. Written informed consent was obtained from all the patients.

\section{Patient consent for publication}

Not applicable.

\section{Competing interests}

LZ, GS, LL, YY and GC are employees of Beijing Novogene Bioinformatics Technology Co., Ltd. The authors declare that they have no competing interests.

\section{References}

1. Zeinalian M, Hashemzadeh-Chaleshtori M, Salehi R and Emami MH: Clinical aspects of microsatellite instability testing in colorectal cancer. Adv Biomed Res 7: 28, 2018.

2. Popat S, Hubner R and Houlston RS: Systematic review of microsatellite instability and colorectal cancer prognosis. J Clin Oncol 23: 609-618, 2005. 
3. Ribic CM, Sargent DJ, Moore MJ, Thibodeau SN, French AJ, Goldberg RM, Hamilton SR, Laurent-Puig P, Gryfe R, Shepherd LE, et al: Tumor microsatellite-instability status as a predictor of benefit from fluorouracil-based adjuvant chemotherapy for colon cancer. N Engl J Med 349: 247-257, 2003.

4. Le DT, Uram JN, Wang H, Bartlett BR, Kemberling H, Eyring AD, Skora AD, Luber BS, Azad NS, Laheru D, et al: PD-1 blockade in tumors with mismatch-repair deficiency. N Engl J Med 372: 2509-2520, 2015.

5. Weinberg BA, Xiu J, Hwang JJ, Shields AF, Salem ME and Marshall JL: Immuno-oncology biomarkers for gastric and gastroesophageal junction adenocarcinoma: Why PD-L1 testing may not be enough. Oncologist 23: 1171-1177, 2018.

6. Boland CR and Goel A: Microsatellite instability in colorectal cancer. Gastroenterology 138: 2073-2087.e2073, 2010.

7. Kelkar YD, Strubczewski N, Hile SE, Chiaromonte F, Eckert KA and Makova KD: What is a microsatellite: A computational and experimental definition based upon repeat mutational behavior at A/T and GT/AC repeats. Genome Biol Evol 2: 620-635, 2010.

8. Müller A, Giuffre G, Edmonston TB, Mathiak M, Roggendorf B Heinmöller E, Brodegger $T$, Tuccari G, Mangold E, Buettner R, et al: Challenges and pitfalls in HNPCC screening by microsatellite analysis and immunohistochemistry. J Mol Diagn 6: 308-315, 2004

9. Hempelmann JA, Lockwood CM, Konnick EQ, Schweizer MT, Antonarakis ES, Lotan TL, Montgomery B, Nelson PS, Klemfuss N, Salipante SJ and Pritchard CC: Microsatellite instability in prostate cancer by PCR or next-generation sequencing. J Immunother Cancer 6: 29, 2018.

10. Roychowdhury S, Iyer MK, Robinson DR, Lonigro RJ, Wu YM, Cao X, Kalyana-Sundaram S, Sam L, Balbin OA, Quist MJ, et al Personalized oncology through integrative high-throughput sequencing: A pilot study. Sci Transl Med 3: 111ra121, 2011.

11. Uzilov AV, Ding W, Fink MY, Antipin Y, Brohl AS, Davis C, Lau CY, Pandya C, Shah H, Kasai Y, et al: Development and clinical application of an integrative genomic approach to personalized cancer therapy. Genome Med 8: 62, 2016.

12. Huang MN, McPherson JR, Cutcutache I, Teh BT, Tan P and Rozen SG: MSIseq: Software for assessing microsatellite instability from catalogs of somatic mutations. Sci Rep 5: 13321, 2015

13. Nowak JA, Yurgelun MB, Bruce JL, Rojas-Rudilla V, Hall DL, Shivdasani P, Garcia EP, Agoston AT, Srivastava A, Ogino S, et al: Detection of mismatch repair deficiency and microsatellite instability in colorectal adenocarcinoma by targeted next-generation sequencing. J Mol Diagn 19: 84-91, 2017.

14. StadlerZK, BattaglinF,MiddhaS, Hechtman JF, Tran C,Cercek A Yaeger R, Segal NH, Varghese AM, Reidy-Lagunes DL, et al: Reliable detection of mismatch repair deficiency in colorectal cancers using mutational load in next-generation sequencing panels. J Clin Oncol 34: 2141-2147, 2016.

15. Salipante SJ, Scroggins SM, Hampel HL, Turner EH and Pritchard CC: Microsatellite instability detection by next generation sequencing. Clin Chem 60: 1192-1199, 2014.
16. Kautto EA, Bonneville R, Miya J, Yu L, Krook MA, Reeser JW and Roychowdhury S: Performance evaluation for rapid detection of pan-cancer microsatellite instability with MANTIS. Oncotarget 8: 7452-7463, 2017.

17. Li H and Durbin R: Fast and accurate short read alignment with Burrows-Wheeler transform. Bioinformatics 25: 1754-1760, 2009.

18. Li H, Handsaker B, Wysoker A, Fennell T, Ruan J, Homer N, Marth G, Abecasis G and Durbin R; 1000 Genome Project Data Processing Subgroup: The sequence alignment/map format and SAMtools. Bioinformatics 25: 2078-2079, 2009.

19. Boland CR, Thibodeau SN, Hamilton SR, Sidransky D, Eshleman JR, Burt RW, Meltzer SJ, Rodriguez-Bigas MA, Fodde R, Ranzani GN and Srivastava S: A National Cancer Institute Workshop on Microsatellite Instability for cancer detection and familial predisposition: Development of international criteria for the determination of microsatellite instability in colorectal cancer. Cancer Res 58: 5248-5257, 1998.

20. Grady WM and Carethers JM: Genomic and epigenetic instability in colorectal cancer pathogenesis. Gastroenterology 135 1079-1099, 2008.

21. Vilar E and Gruber SB: Microsatellite instability in colorectal cancer-the stable evidence. Nat Rev Clin Oncol 7: 153-162, 2010.

22. Bacher JW, Flanagan LA, Smalley RL, Nassif NA, Burgart LJ, Halberg RB, Megid WM and Thibodeau SN: Development of a fluorescent multiplex assay for detection of MSI-high tumors. Dis Markers 20: 237-250, 2004.

23. Middha S, Zhang L, Nafa K, Jayakumaran G, Wong D, Kim HR, Sadowska J, Berger MF, Delair DF, Shia J, et al: Reliable pan-cancer microsatellite instability assessment by using targeted next-generation sequencing data. JCO Precis Oncol 2017: 2017.

24. Umar A, Boland CR, Terdiman JP, Syngal S, de la Chapelle A, Rüschoff J, Fishel R, Lindor NM, Burgart LJ, Hamelin R, et al: Revised Bethesda guidelines for hereditary nonpolyposis colorectal cancer (Lynch syndrome) and microsatellite instability. J Natl Cancer Inst 96: 261-268, 2004.

25. Hempelmann JA, Scroggins SM, Pritchard CC and Salipante SJ: MSIplus for integrated colorectal cancer molecular testing by next-generation sequencing. J Mol Diagn 17: 705-714, 2015.

26. de la Chapelle A and Hampel $\mathrm{H}$ : Clinical relevance of microsatellite instability in colorectal cancer. J Clin Oncol 28: 3380-3387, 2010.

27. Hause RJ, Pritchard CC, Shendure J and Salipante SJ: Classification and characterization of microsatellite instability across 18 cancer types. Nat Med 22: 1342-1350, 2016.

28. Pawlik TM, Raut CP and Rodriguez-Bigas MA: Colorectal carcinogenesis: MSI-H versus MSI-L. Dis Markers 20: 199-206, 2004. 